\title{
Parallel Algorithm for the Voltage Profile Simulation for the Synthesis of Superhard Materials Under High Pressure and High Temperature
}

\author{
Dalessandro Soares Vianna \\ Federal Fluminense University (UFF), Rio das Ostras, RJ, Brazil
}

\author{
João José de Assis Rangel \\ Candido Mendes University (UCAM), Campos dos Goytacazes, RJ, Brazil
}

Sicilia Ferreira Ponce Pasini Judice

National Laboratory for Scientific Computing (LNCC), Petrópolis, RJ, Brazil

Marcilene de Fátima Dianin Vianna

Federal Fluminense University (UFF), Campos dos Goytacazes, RJ, Brazil

\begin{abstract}
This article shows a parallel algorithm developed to simulate the electric voltage profile applied to the heating of the compression chamber used in the process of superhard materials synthesis, specifically, diamonds. The computational simulation for the determination of the distribution of the eletrical potential (d.d.p. in volt) is necessary to permit to get, with precision, the value of the heat (in W/mm) that is generated by the eletric current flux in the process. The analysis of temperature distribution let us investigate the graphite to diamond transformation process and also get diamonds of better quality and lower cost. The computational simulation is an important tool in the superhard materials syntesis research, once the real tests show a very high cost and many times they do not show satisfactory answers. We hope we can contribute with the techniques of computational simulation for the diamond synthesis process, using resources of parallelism shown in this article. The purpose to the use of paralelism techniques in the computational simulations is situated in the possibility of offering an ampler research of the superhard material synthesis process, through enlargement of the volume studied in a time of the reduced processing. In the simulations carried out in a small part of the compression chamber of $10 \mathrm{~mm}$ of ray per $10 \mathrm{~mm}$ of height, using a matrix $100 \times 100$, it was necessary nearly one hour of processing in a SUN FIRE machine with one SPARC III $750 \mathrm{MHz}$ processor. A completed chamber, used in the diamond synthesis, has measurements from $60 \mathrm{~mm}$ to $100 \mathrm{~mm}$ and that can be enlarged if there is interest in analysing other parts of the high pressure apparatus that permit the coupling of the compression chamber to the hydraulic press.
\end{abstract}

Keywords: Parallel algorithm, Finite difference method, Diamond synthesis. 


\section{Introduction}

Diamonds are materials chemically inert, with high hardness, low friction coefficient, high thermic conductivity and an excellent electrical insulator. These attractive physical chemistry qualities make them essential in the cutting tools production, that goes through constant wear and tear. The diamond is in almost all lines of the industry, from the metal-mechanic up to the production of wood. The technology control for the production of synthetic diamond can put Brazil into the small group of countries that has this capability of production.

The production techniques of synthetic diamonds started in the fifties. The first oficial synthesis carried out by General Electric happened in 1954. In Brazil, 1987, researchers from Rio Grande do Sul University registered the first diamond synthesis. From 2001 until nowadays, the Norte Fluminense University has already produced ten thousand carats of diamonds, around two kilogramme, using the technique of high temperature and high pressure production.

The methods based on the graphyte to diamond transformation, in static high pressure (4.3 GPa to $8.0 \mathrm{GPa}$ ) and temperature $(1450 \mathrm{~K}$ to $2100 \mathrm{~K}$ ) use different compression chambers (1 GPa equals $10.000 \mathrm{~atm}$.). These chambers provide the necessary high presssures and temperatures to the synthesis process, but they also cause strong temperature and pressure gradients in the process. The temperature gradients are undesirable, because they influence negatively the characteristics of the produced diamonds and also the lifetime of some equipments (Bundy et al., 1961; Bundy, 1995).

The direct measurement of the temperature into the process is very limited, because of the severe conditions existent in the process (the solid corps become plastically deformed) and also because of constructive characteristics (the compression chamber stays into a metal apparatus of high hardness that makes the coupling to the hydraulic press). The heating of the process is done through the passage of electric current through the compressed corps (the graphite itself that will become into diamond). The electric voltage applied to the compressed corps (graphite and catalist metal arranged in different composition) causes the generation of heating and, the temperature controls are done through the voltage control applied to the process.

The voltage profile applied for heating depends on the constructive arrangement and on the properties of the present elements into the apparatus that generate the high pressure (graphite, catalist metal, etc.) at the beginning of the synthesis. This same electrical voltage profile will influence the way how the graphite to diamond transformation will happen, since the temperature distribution is dependent on the voltage profile, among other effects related also to the constructive arrangements and properties of the present elements of the synthesis beginning.

Analysing constructive alternatives, that can minimize the temperature gradients, they can result in the obtainment of diamonds with better quality and lower cost. It can be emphazised that the cost of this research, if done only in an experimental way (in laboratory), is very expensive. Another characteristic of this process is that fifty per cent of the synthetic diamond cost is related to the cost of the generated apparatus of high pressure and temperatures and the lifetime of the apparatus is limited to one hundred operations at most. All these factors put the development of the manufacture technologies and the raise of lifetime of these apparatuses in the core of the scientific matter about the diamonds synthesis. 
It can be possible to study with the help of computational simulation, in a feasible way, the temperature and voltage ditribution, both in the synthesis region (compression chamber) and in the high pressure apparatus. It is possible to investigate the temperature gradients, constructive variations of the high pressure apparatus compounds and reactive cell, variation of the synthesis compounds properties, etc., through the computational simulation.

Reducing the processing time and allowing the execution of the computational simulation is a strategy of impact to support the research and development of diamond synthesis technology. The use of parallel strategies is a good way to reduce the computational time (Drummond et al., 2001; Ochi et al., 1999; Vianna et al., 1999, 2007,2010 ). A lot of papers have been published about computational simulation in the research of superhard materials synthesis until now (Levitas et al., 1989; Novikov et al., 1991; Rangel et al., 1998), however the application of parallelism techniques is still a novelty in this area.

The organization of the paper is described as following. In the next section, it is described fhe high pressure apparatus. In Section 3, it is presented the modelling problem. The parallel algorithm is presented in Section 4. The computational results are presented in Section 5. Finally, Section 6 contains the concluding remarks.

\section{Description of the High Pressure Apparatus}

Figure 1 shows the diagram used in making calculations for high pressure apparatus diagram, used in a $2500 \mathrm{t}$ press, before and during the compression. This

(a)
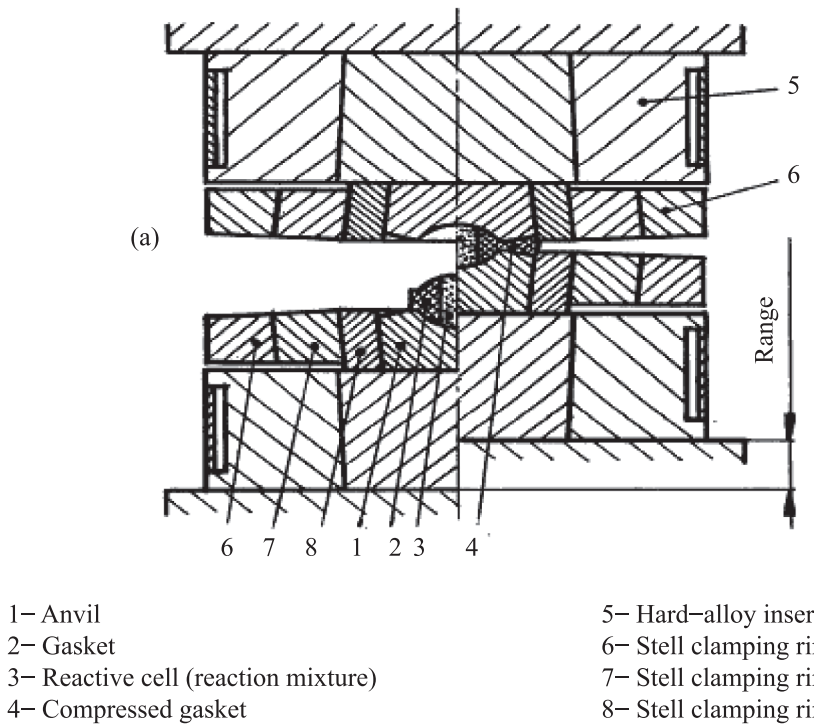

5- Hard-alloy insert in thrust plate 6- Stell clamping rings - external

7- Stell clamping rings - medium 8- Stell clamping rings - internal

Figure 1. Diagram used in making calculations for high pressure apparatus of anvil type recesses (a- before compress; b- after compress). 1-Anvil; 2-Gasket; 3 - Reactive cell (reaction mixture); 4-Compressed gasket; 5 - Hard-alloy insert in thrust plate; 6 - Stell clamping rings - external; 7 - Stell clamping rings - medium; 8 - Stell clamping rings - internal. 
assemblage, $2500 \mathrm{t}$ press and high pressure apparatus, can generate pressure and temperature near $5 \mathrm{GPa}$ and $1700 \mathrm{~K}$, respectively.

Figure 2 shows the scheme of the central part of the high pressure apparatus in the compressed state. It can be observed the following components in this figure: anvil, gasket and reactive cell. The reactive cell is the place where occurs the graphite to diamond transformation. It is the exact place of the synthesis reaction. Analysing Figure 2, it can be noticed that the reactive cell stays thermically insulated only in the gasket side, that has low thermical conductivity coefficient. The thermical insulation is low and the heat is dispersed in high value in the superior and inferior sides of the reactive cell. These parts are in contact with the anvil, constituted of high hardness metal and high mechanical strength, with high thermical conductivity coefficient. This constructive characteristic causes high temperature gradients in the reactive cell and in the anvil part that is in direct contact with it.

Constructive variations of this apparatus can minimize the temperature gradients, both in the reactive cell and in the anvil (Levitas et al., 1989; Novikov et al., 1991). The temperature gradients in the reactive cell influence the characteristics of the produced diamonds, however the gradients in the anvil influence lifetime itself. The region of the anvil in contact with the reactive cell is subdued to highest gradients and values of temperatures, being this the most critic region of this part (Poliakov et al., 1990, 1996).

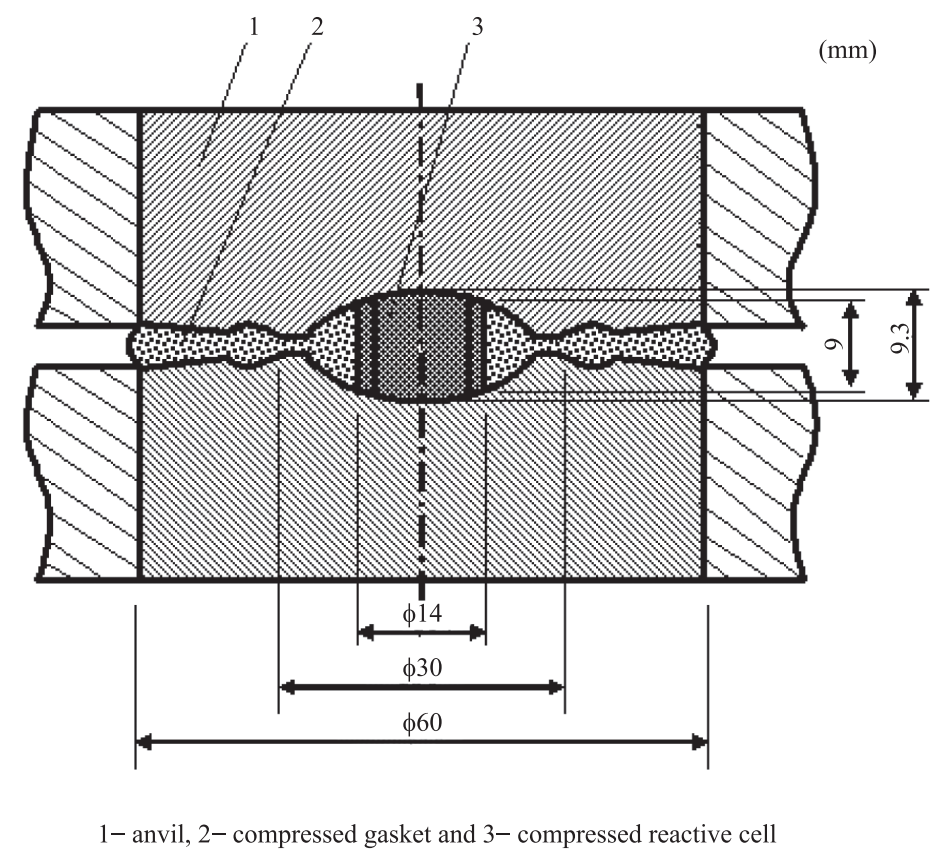

Figure 2. Detail of the high pressure apparatus of anvil type toroidal (compression chamber: gasket and reactive cell in the compressed state). 1-Anvil, 2 - Compressed gasket and 3 -Compressed reactive cell. 


\section{Modelling Problem}

The model approaches just one side of the compression chamber because of the cilindric simetry. Figure 3 shows in a grid form the focused parts of compression chamber decribed in Figure 2. Observing the figure, it can be noticed that it is necessary to extend the matrix to extend the part of the analysed object for keeping the value precision, on the other hand, it is important to extend the number points of the matrix in a small region of the chamber for increasing the value precision.

It was used the differential equation of heat conduction in a stationary medium and two spatial co-ordinates that, for bodies of revolution the cilindrical-polar system, will be used with $x$ denoting axial co-ordinate and $y$ the radial one, described below with the respective boundary conditions (Gosman et al., 1985).

$$
\frac{\partial}{\partial x}\left(k \cdot x \frac{\partial V}{\partial x}\right)+\frac{\partial}{\partial y}\left(k \cdot x \frac{\partial V}{\partial y}\right)=0
$$

Figure 4 presents the boundary conditions, where $h$ and $L$ are the height and ray at the high pressure chamber, respectively. $V_{0}$ is na applied voltage and $k$ is the conductive coefficient.

It was used the technique of the finite difference with explicit iterative with Tchebychev parameter scheme to the numerical solution (Samarskii and Vabishchevich, 1996). In the literature related, for problems like these, the most useful methods are the finite difference methods (FDM) and the finite element methods (FEM). Both techniques are used with advantages and disadvantages in cases of numerical modelling problems of voltage and temperature distribution. On the present case, there is a phase transformation where the FDM is more useful.

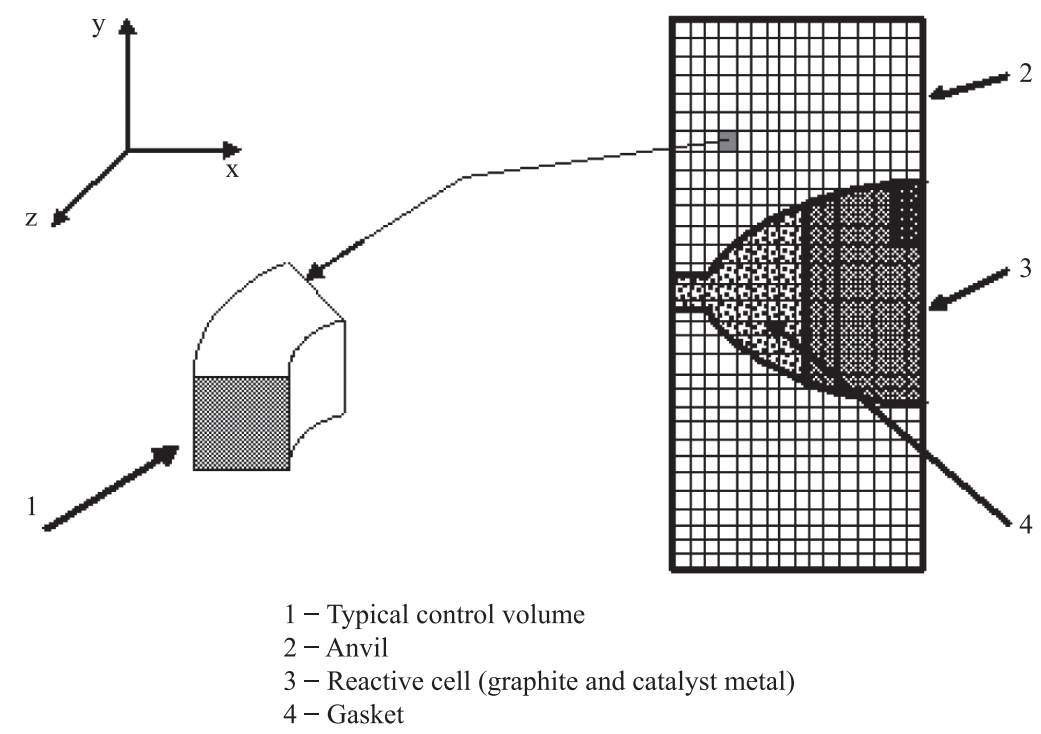

Figure 3. A typical computational grid and associated control volumes. 1 - Typical control volume; 2 -Anvil; 3 - Reactive cell (graphite and catalyst metal); 4 - Gasket. 


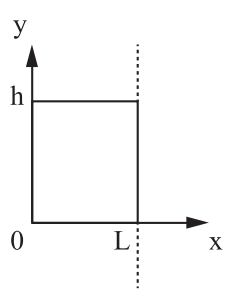

$$
\begin{aligned}
& V=V(x, y) \\
& V=V(x, 0)=0 \\
& \left.\frac{\partial V}{\partial x}\right|_{x=0}=0
\end{aligned}
$$$$
k=k(x, y)
$$$$
V(x, h)=V_{0}
$$

(simmetry condition)

Figure 4. Boundary conditions.

In finite difference form the Equation 1 is written as below

$$
\frac{\partial}{\partial x_{1}}\left(k_{1}\left(x_{1}, x_{2}\right) \frac{\partial u}{\partial x_{1}}\right)+\frac{\partial}{\partial x_{2}}\left(k_{2}\left(x_{1}, x_{2}\right) \frac{\partial u}{\partial x_{2}}\right)=0
$$

The solution for this problem is then given by

$$
y_{i j}^{(k+1)}=y+i j^{(k)}-\tau_{k+1} r_{i j}^{(k)}, i=1,2, \ldots, N_{1}-1, j=1,2, \ldots, N_{2}-1 .
$$

where:

$$
\begin{aligned}
& r_{i j}^{(k)}=-\left(a_{1} y \frac{(k)}{x_{1}}\right)_{x_{1}, i j}-\left(a_{2} y \frac{(k)}{x_{2}}\right)_{x_{2}, i j}, \\
& \left(a_{1} y_{x_{1}}\right)_{x_{1}, i j}=\frac{1}{h_{1}}\left[a_{1, i+1, j}\left(y_{i+1, j}-y_{i j}\right) / h_{1}-a_{1, i j}\left(y_{i j}-y_{i-1, j}\right) / h_{1}\right], \\
& \left(a_{2} y_{x_{2}}\right)_{x_{2}, i j}=\frac{1}{h_{2}}\left[a_{2, i, j+1}\left(y_{i, j+1}-y_{i j}\right) / h_{2}-a_{2, i j}\left(y_{i j}-y_{i, j-1}\right) / h_{2}\right], \\
& i=1,2, \ldots, N_{1}-1, j=1,2, \ldots, N_{2}-1, \\
& \tau_{k} \text { is a correction factor, } \\
& N_{1} \text { and } N_{2} \text { are the number of lines and columns, respectively. }
\end{aligned}
$$

\section{Proposed Parallel Algorithm}

The proposed parallel strategy is based on the pipeline technique (Silberschatz and Galvin, 1997). First of all, the matrix $y$ is divided (horizontal cut) among the $p$ processors, as shown on Figure 5a. As seen on Section Modelling Problem (Equation 3), the calculus of each $y_{i, j}$ element, besides using two elements of the same line $\left(y_{i, j-1}\right.$ and $\left.y_{i, j+1}\right)$, uses an element of the previous line $\left(y_{i-1, j}\right)$ and an element of the posterior line $\left(y_{i+1, j}\right)$, where $1 \leq i \leq N_{1}$ and $1 \leq j \leq N_{2}$. This dependence of elements of other lines causes a problem in the frontier regions. On Figure $5 b$, the frontiers are represented by lines in gray. In these regions, a processor $P_{t}$ needs an element of the processor $P_{t-1}$, in case it is the first line of the submatrix $y_{t}$ (piece of the $y$ matrix related to the processor $P_{t}$ ), or an element of the process $P_{t+1}$, in case it is the last line. 
(a)
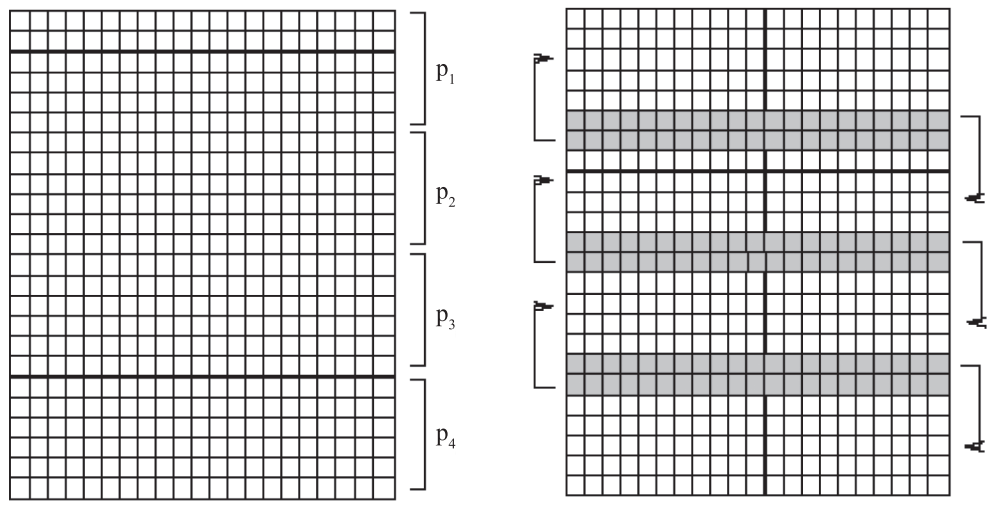

Figure 5. Proposed parallelism strategy.

The proposed algorithm is started with the process $P_{1}$ carrying out the first iteration about the submatrix $y_{1}$. When $P_{1}$ ends the first iteration, this one sends the last line of $y_{1}$ to the process $P_{2}$ so that this one can start its first iteration. $P_{2}$ starts the first iteration while, at the same time, $P_{1}$ starts its second iteration. The process $P_{2}$ calculates the first line of $y_{2}$ and sends it to the process $P_{1}$ because this one will be necessary when $P_{1}$ calculates the last line of $y_{1} . P_{2}$ goes on the processing of the first iteration and when it is finished, the last line of $y_{2}$ is sent to the process $P_{3}$ and $P_{2}$ starts its second iteration, while at the same time $P_{3}$ starts its first and $P_{1}$ is already starting its third. This procedure repeats until the last process $P_{p}$ finishes its iteration of number $n$, where $p$ and $n$ are algorithm input parameters and represent, respectively, the number of processors and the total number of iterations.

In the pipeline technique used in operational systems (Silberschatz and Galvin, 1997), if there are $Z$ jobs to be executed and each one of them has $z$ tasks (considering that each of these tasks is executed in one unit of time), the first job is executed in $z$ units of time. The second job uses $z-1$ units of time of the previous job and needs only one additional unit of time. This happens with all of the others jobs. So, the expected time for the conclusion of the $Z$ jobs is of

Total Time $=z+(Z-1)=Z+z-1$.

If there are a number of jobs much higher than the number of tasks $(Z>>z)$, it can be told that

Total Time $\approx Z$.

The ideal speedup in a pipeline with $z$ tasks and $Z$ jobs is:

Speedup $=\frac{Z \times z}{Z}=z$

In analogy with the parallel strategy adopted in this paper it can be told that the ideal speedup for a system with $p$ processes carrying out $n$ iterations $(n>>p)$ is:

$$
\text { Speedup }=\frac{n \times p}{n}=p
$$




\section{Computational Results}

The computational experiments were carried out in a SUN FIRE 6800 with SPARC III 750MHZ processors and 24Gb RAM.

The parallel algorithm proposed was implemented using the $\mathrm{C}$ programming language and MPI library for the parallelism.

In the first experiment, it was used a matrix $100 \times 100$ simulating a piece of the chamber of $10 \mathrm{~mm}$ of ray per $10 \mathrm{~mm}$ of height. The number of iterations $(n)$ carried out was enough for having the convergence of the result, $n=10000000$ (tem milions).

Table 1 shows, to $1 \leq p \leq 4$ processes, the obtained time and the speedup. The speedup achieved with $p$ processes is calculated dividing the time spent by the algorithm using one process $\left(t_{1}\right)$ divided by the time obtained $\left(t_{p}\right)$.

This experiment was done with $1 \leq p \leq 4$ processes because with $p>4$ processes, the algorithm started losing efficiency (Ex.: $t_{5}>t_{4}$ ). That can be justified because according to the number of processes increases, the region of the matrix $y$ subordinated to each process decreases. As that region decreases, the carried out processing becomes smaller, making that the onus caused by the comunication starts influencing more and more in the total time of the algorithm.

In the second experiment, it was used a matrix $600 \times 600$ simulating a cylinder of $60 \mathrm{~mm}$ of ray per $60 \mathrm{~mm}$ of height. The number of iteration was fixed in $n=1000$ ( 1 thousand). The purporse of this test was to verify the performance of the proposed parallel algorithm using $1 \leq p \leq 8$ processes, since a matrix of bigger dimension was being used.

Table 2 shows for $1 \leq p \leq 8$ processes, the time obtained and the speedup. The reduction of the processing time with the increase of the number of process can be better seen on Figure 6 .

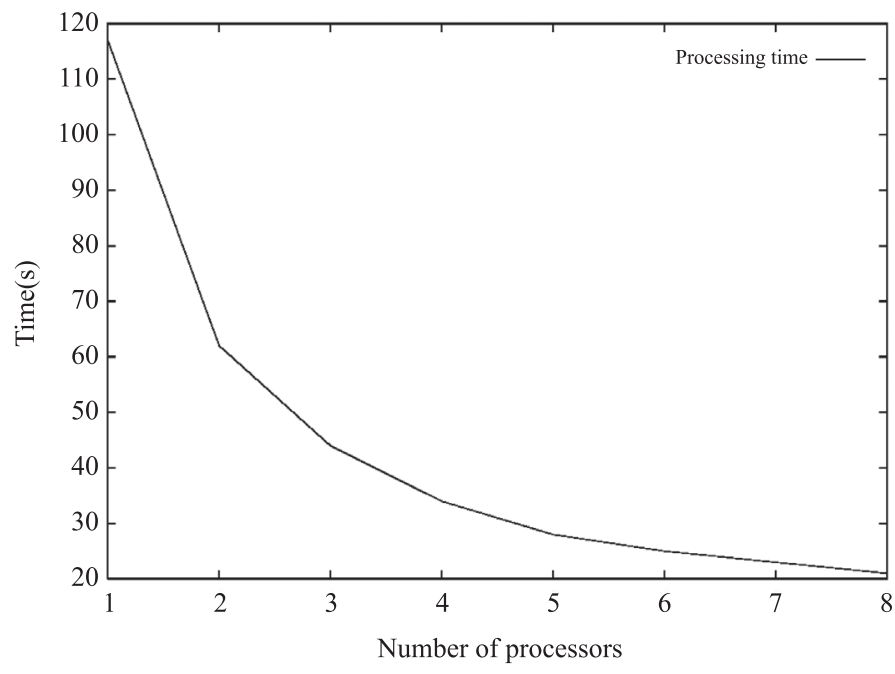

Figure 6. Processing time $\mathrm{X}$ number of processors. 
Table 1. Results obtained with a matrix $100 \times 100$.

\begin{tabular}{|c|c|c|}
\hline$p$ & Time(s) & Speedup \\
\hline 1 & 29714 & 1.00 \\
\hline 2 & 15481 & 1.92 \\
\hline 3 & 10487 & 2.83 \\
\hline 4 & 8006 & 3.71 \\
\hline
\end{tabular}

Table 2. Results obtained with a matrix $600 \times 600$.

\begin{tabular}{|c|c|c|}
\hline$p$ & Time(s) & Speedup \\
\hline 1 & 117 & 1.00 \\
\hline 2 & 62 & 1.89 \\
\hline 3 & 44 & 2.66 \\
\hline 4 & 34 & 3.44 \\
\hline 5 & 28 & 4.18 \\
\hline 6 & 25 & 4.68 \\
\hline 7 & 23 & 5.09 \\
\hline 8 & 21 & 5.57 \\
\hline
\end{tabular}

\section{Conclusion}

The simulation process analysed demands a very high computational effort. According to the dimentions of the region under analysis increases, that effort increases exponentially. The experiments made show that applying parallelism in this process can reduce a lot the processing total time.

The experiments also showed that the more the region analysed increases, the more the gain with the parallelism, caused by the use of a high number of processors.

\section{Acknowledgements}

This work was funded by: Conselho Nacional de Desenvolvimento Científico e Tecnológico (CNPq) and Fundação de Amparo à Pesquisa do Estado do Rio de Janeiro (FAPERJ). The computational experiments were done at the Laboratório Nacional de Computação Científica - LNCC.

\section{References}

Bundy, F.P.; Bovenkerk, H.P.; Strong, H.M. and Wentorf, R.H. (1961) DiamondGraphite Equilibrium Line from Growth and Graphitization of Diamond. Journal of Chemical Physics, Vol. 35, No. 2, pp. 383-391. http://dx.doi.org/10.1063/1.1731938

Bundy, F.P. (1995) The Pressure-Temperature Phase and Reaction Diagram for Carbon - Mechanical Behavior of Diamond and other Forms of Carbon. Materials Research Symposium, Vol. 383, pp. 3-20. 
Drummond, L.M.A.; Ochi, L.S. and Vianna, D.S. (2001) An asynchronous parallel metaheuristic for the period vehicle routing problem. Future Generation Computer Systems, Vol. 17, pp. 379-386. http://dx.doi.org/10.1016/S0167-739X(99)00118-1

Gosman, A.D.; Launder, B.E. and Reece, G.J. (1985) Computer-Aided Engineering Heat Transfer and Fluid Flow. Nova York: John Wiley \& Sons.

Levitas, V.I.; Idesman, A.V.; Leshchuk, A.A. and Polotnyak, S.B. (1989) Numerical Modeling of Thermomechanical Processes High Pressure Apparatus Applied for Superhard Materials Synthesis. High Pressure Science and Technology, pp. 38-40.

Novikov, N.V.; Levitas, V.I.; Leshchuk, A.A. and Idesman, A.V. (1991) Mathematical Modeling of Diamond Synthesis Process. High Pressure Research, Vol. 7, pp. 195-197. http:// dx.doi.org/10.1080/08957959108245544

Ochi, L.S.; Vianna, D.S.; Drummond, L.M.A. and Victor, A.O. (1999) A parallel evolutionary algorithm for the vehicle routing problem with heterogeneous fleet. Future Generation Computer Systems, Vol. 14, pp. 285-292. http://dx.doi.org/10.1016/S0167-739X(98)00034-X

Poliakov, V.P.; Elyuntin, V.P.; Terent'ev, S.A. and Mourachov, S. (1990) Effect of Geometry of the Growth System on the Morphology of Diamond Crystal. Soviet Physics - Doklady, Vol. 35, No. 4, pp. 376-277.

Poliakov, V.P.; Potemkin, A.A. and Monteiro, S.N. (1996) Estabilidade e Resistência de Diamantes Sintéticos do Tipo Carbonado, in: $12^{\circ}$ Congresso Brasileiro de Engenharia e Ciência dos Materiais, São Paulo, Vol. 1, pp. 222-225.

Rangel, J.J.A.; Mourachov, S. and Poliakov, V.P. (1998) Analysis of Voltage and Temperature Distribution for Polycrystalline Diamond Synthesis. High Pressure Sci. Technol, Vol. 7, pp. 1007-1009. http://dx.doi.org/10.4131/jshpreview.7.1007

Samarskii, A.A. and Vabishchevich, P.N. (1996) Computational Heat Transfer: The Finite Difference Methodology. Nova York: John Wiley \& Sons.

Silberschatz, A. and Galvin, P.B. (1997) Operating System Concepts. 5th ed. Nova York: John Wiley \& Sons.

Vianna, D.S.; Ochi, L.S. and Drummond, L.M.A. (1999) A parallel hybrid evolutionary metaheuristic for the period vehicle routing problem, in: II workshop on bio-inspired solution to parallel processing problems (BIO'99), Lecture notes in computer science, San Juan, Vol. 1586, pp. 183-191.

Vianna, D.S.; Arroyo, J.E.C.; Vieira, P.S. and Azeredo, T.R. (2007) Parallel strategies for a multi-criteria GRASP algorithm. Produção, Vol. 17, pp. 1-12. http://dx.doi.org/10.1590/ S0103-65132007000100006

Vianna, D.S.; Robl, S.R.C. and Dianin, M.F. (2010) A GRASP+VND algorithm for a class of job scheduling problem in parallel machines. Brazilian Journal of Operations and Production Management, Vol. 7, pp. 63-69.

\section{Biography}

Dalessandro Soares Vianna is a Computer Science Professor of Federal Fluminense University. He holds a BS in Computer Science from Federal Fluminense University (1996), Master in Computer Science from Federal Fluminense University (1999) and Ph.D. in Computer Science from Catholic University of Rio de Janeiro (2004). His research interests include Operational Research, Metaheuristics and Parallel Programming.

Contact: dalessandrosoares@yahoo.com.br 
João José de Assis Rangel is a Professor of Candido Mendes University and Federal Fluminense Institute. He holds a BS in Mechanical Engineering from Gama Filho University (1991), Master in Mechanical Engineering from Military Institute of Engineering (1994) and Ph.D. in Material Engineering from Norte Fluminense University (1998). His research interests include Computational Simulation, Operational Research and Diamonds Synthesis.

Contact: joao@ucam-campos.br

Sicilia Ferreira Ponce Pasini Judice holds a BS in Computer Science from Candido Mendes University (2006) and Master in Computational Modeling from Computer Science National Laboratory (2009). She is a Ph.D. student in Computational Modeling from Computer Science National Laboratory. Her research interests include Computational Simulation, Graphic Computation and Computational Modeling.

Contact: siciliajudice@gmail.com

Marcilene de Fátima Dianin Vianna is a Mathematics Professor of Federal Fluminense University. She holds a BS in Mathematics from University of Maringá (1998) and Master in Mathematics from Catholic University of Rio de Janeiro (2001). She is a Ph.D. student in Natural Sciences from Norte Fluminense University. Her research interests include Applied Mathematics, Metabolomics, Operational Research and Signal Processing.

Contact: marcilenedianin@gmail.com

\section{Article Info:}

Received: May, 2010

Accepted: July, 2012 